\title{
EL RETO DE LOS AÑOS NOVENTA: SUS LOGROS, DIFICULTADES Y SUEÑOS EN UN CONTEXTO DE CRISIS PRESUPUESTARIA PARA QUIENES NO ÉRAMOS PRIORIDAD EN LA UNIVERSIDAD
}

\author{
Eddie Alberto Vargas Rodríguez ${ }^{1}$ \\ Jubilado de la Universidd Nacional \\ Heredia, Costa Rica
}

Recibido 8 de noviembre 2007 • Aprobado 30 de enero 2008

Después de muchos años de servicio en la academia universitaria de la Universidad Nacional, institución a la que acompañé en su desarrollo desde diferentes perspectivas: en el aula, en la comunidad, en la administración y en la investigación, a partir de junio del año 1992, enfrenté el reto de conducir el CIDE y de tratar de realizar mis sueños profesionales en compañía de muchos amigos, amigas, compañeras y compañeros de la academia, que creyeron en mi persona y mis ideales. Algunos compartíamos esos sueños, otros tenían proyectos distintos; pero en fin, el propósito era común: construir positivamente para hacer del Centro, la Universidad y la Educación un proyecto de vida.

Mis sueños y realidades se fundamentaban en principios que orientaron mi quehacer; entre ellos consideré, y sigo considerando, que la Educación es un compromiso de todos, en el que debe prevalecer el respeto entre sus actores, donde nadie se impone ni domina a los otros, donde se fomenta la unidad en la diversidad, donde discrepar se considera importante para crecer y aprender de la otra persona.

Por ello, mi compromiso era impulsar la Educación como un medio para construir una sociedad más humana y libre, para formar seres críticos, creativos y capaces de transformar el mundo. Esos principios inspiraron mi gestión; sin embargo, para hacer verdad ese sueño, mis leales compañeras y compañeros en la construcción del Centro y mi persona, enfrentamos realidades como las que describo más adelante; retos, logros y dificultades que nos permitieron contribuir al desarrollo del Centro, con compromiso humanista, lealtad a los demás y respeto a las ideas adversas.

\section{LA REALIDAD QUE ENFRENTAMOS}

La Universidad Nacional, en los primeros años de esta gestión, vivió una seria crisis presupuestaria que afectó seriamente el desarrollo académico; en este ambiente, la principal solución ofrecida por la

\footnotetext{
Actualmente es profesor jubilado de la Universidad Nacional (UNA). Fue decano del Centro de Investigación y Docencia en Educación (CIDE) en el periodo 1992-1998.
} 
administración central era el recorte de plazas y el cierre de proyectos. Esos fueron los tiempos de las famosas "rondas presupuestarias": la Rectoría se reunía con las autoridades de cada facultad o centro para definir cuáles proyectos y carreras dejaba y cuáles recortaba, en virtud de que no había presupuesto, sobre todo de operación; por eso, algunas veces se recurría a transformar plazas en presupuesto de operación, lo que cerraba acciones de la academia. Para hacerlo, se debía solicitar la autorización de la Contraloría de la República, gestión que tomaba mucho tiempo para las carreras de educación, pues no éramos prioridad dentro del desarrollo académico universitario, política no plasmada por escrito, pero demostrada por la práctica de las autoridades universitarias en la administración central...

Mientras tanto, en el país había una gran demanda de educadores, tanto para los niveles de primaria como los de secundaria, dado que los centros formadores no graduaban el personal necesario para satisfacer las demandas del sistema educativo. En secundaria, las áreas más deficitarias eran: Ciencias Naturales, Español, Estudios Sociales, Matemática e Inglés. La solución ofrecida por el Ministerio de Educación Pública (MEP) consistió en financiarles a las universidades públicas esa formación, mediante un préstamo de los bancos internacionales, acción que se ejecutó mediante el convenio UNIVERSIDADES, MEP, BID, BIRF, y que coordinó la Comisión de Decanos y Decanas del CONARE y la viceministra de Educación del gobierno de turno.

Cabe destacar que al interior de la Universidad, los recursos eran administrados desde dos oficinas: Educación Secundaria tenía una oficina propia, adscrita a la Vicerrectora de Docencia, que giraba los recursos a la División de Educología y las escuelas respectivas de las otras facultades para el desarrollo de los componentes pedagógico en el CIDE y específicos de las carreras de enseñanza del Inglés, Español, Ciencias Naturales, Estudios Sociales y Matemática, las cuales se impartían en Heredia, Ciudad Neily, Puriscal, Pérez Zeledón y Liberia. Por su parte la Educación Primaria era dirigida desde el Decanato del CIDE y el programa del convenio era ejecutado por la División de Educación Rural, con un modelo de entrega presencial y a distancia, y la División de Educación Básica, con el modelo presencial. Esta situación nos exigió ajustar la duración de los planes de estudio, por las características que tiene la entrega de la docencia. Los planes fueron ejecutados en San Carlos, Puriscal, Cañas, Liberia, Nicoya, Pérez Zeledón y en la sede central de Heredia.

Al Decanato le correspondía velar por el desarrollo de los planes, tanto de secundaria como de primaria, además negociar la cantidad de grupos que se asumían cada año, intervenir en los problemas que generaba la admisión y facilitar las acciones para garantizar planes de estudio similares entre la UNED, la Universidad de Costa Rica y la UNA, para garantizar la movilidad de los estudiantes entre la tres universidades. Lo anterior exigió elaborar tablas de reconocimiento de estudios entre las universidades, reuniones para diseñar, en conjunto la carrera, acción que tuvo mucho éxito en las carreras de educación primaria.

Además de ese proceso, la Decanatura se vio involucrada en planes de estudio de posgrado que, financiados por el Convenio, favorecieran a los académicos del CIDE. Esta acción permitió que cinco docentes fueran becados para estudiar Evaluación en la Universidad de Costa Rica. Al mismo tiempo, la Decanatura tuvo que asumir programas de capacitación para docentes en servicio, también financiados por el Convenio.

Como puede observarse, esa fue una de las áreas de trabajo que demandó gran parte de nuestro esfuerzo: También trabajamos intensamente en investigación, extensión, docencia y producción, que se desarrollaban en un contexto de crisis, pues en el CIDE esas acciones tenían muy poco espacio: la academia se limitaba la docencia. No obstante, estas áreas se beneficiaron por el desarrollo del Convenio, y les permitió operar porque no había en la Universidad recursos para atender la operación del centro como correspondía. 
Amén del aporte económico para el desarrollo de los planes de estudio en áreas de formación de docentes, se acrecentó la cantidad de personal destacado del MEP, práctica que se había iniciado con otros convenios establecidos con el Ministerio, en años anteriores, en el campo de la formación de maestros, especialmente en la División de Educación Rural. Es decir, personal que era pagado por el MEP pero que se incorporaba a las unidades académicas para impartir lecciones en las carreras que financiaba el Convenio. Esto le permitía al CIDE abrir más grupos y graduar más docentes. Es importante destacar que tal práctica era exclusiva de la UNA, pues las otras universidades no contaron con ese recurso.

El funcionario(a) destacado(a) recibía un sobresueldo del $50 \%$ sobre el salario que tenía en el MEP, y había que contratarlo por un cuarto de tiempo para que tuviera vínculo laboral con la Universidad. Era un buen estímulo salarial para que se incorporaran exclusivamente a cumplir los objetivos de las carreras involucradas, con excelencia y calidad.

Es importante aclarar que esta práctica de destacados no se había estipulado en el documento del Convenio, sino que se sustentaba en acuerdos bilaterales con el ministro de turno, y se negociaban cada año dependiendo de la cantidad de grupos que se abrieran y de las necesidades de las divisiones de Educología, Básica y Rural. Hubo años en que se destacaron hasta 10 personas a tiempo completo, lo cual dio un gran aporte al crecimiento en la formación docente.

\section{LOS RETOS}

En ese momento, el CIDE, en coordinación con las sedes regionales, tenía el gran reto de brindar cobertura al plan de formación de diplomados para la educación primaria, y a los profesorados de educación secundaria. Estos últimos, en conjunto con las unidades académicas respectivas: Escuela de Historia, Escuela de Literatura, Escuela de Biología, Escuela de Matemática.

La formación de docentes de educación primaria correspondía a las divisiones de Educación Básica y de Educación Rural, la primera con una oferta totalmente presencial y, la segunda, con una oferta presencial y a distancia, en diferentes lugares del país, durante los fines de semana.

En la Universidad Nacional, además del problema presupuestario la administración del convenio como un recargo de las funciones del Decanato, se desarrollaba un proceso de reorganización interna que comprendía, entre otras cosas, el paso de semestres a trimestres, lo que obligó a las Facultades y Centros a realizar cambios estructurales y curriculares. Particularmente, en el CIDE, se transformaron sus 23 carreras.

En ese contexto, la gestión enfrentó varios retos, enfrentados gracias al apoyo del personal académico y administrativo, y los estudiantes, quienes además de sus funciones, se comprometieron:

- $\quad$ Fortalecer el modelo de desarrollo académico para garantizar excelencia y calidad en el aporte que se le brinda al Centro, a la Universidad y al país, en materia de Educación.

- Mejorar el clima organizacional del Centro, porque habían discrepancias que hacían perder la unidad de la comunidad académica y administrativa, fundamental para un el trabajo en equipo que garantizara el progreso administrativo y académico.

- Mantener la oferta académica en el campo de la formación de docentes, para los diferentes niveles del sistema educativo, con excelencia de su cobertura.

- Fortalecer la investigación y la extensión desde las unidades académicas, porque el Centro de Investigación carecía de proyectos de esa naturaleza en las unidades académicas, salvo los que impulsaba el Instituto del Niño y la División de Educación Rural. 
- Desarrollar posgrados, pues el CIDE no contaba con esa opción y no había contenido presupuestario para hacerlo.

- Responder académica y administrativamente a la formación y capacitación de docentes, mediante el convenio Universidades/ MEP/BID/BIRF.

- Ampliar la oferta académica con nuevas opciones en el nivel de grado académico.

- Reorganizar el Centro de acuerdo con los cambios generados por el nuevo Estatuto Orgánico de la Universidad.

- Replantear la oferta curricular para ajustarla al cambio de semestres a trimestres.

- Ampliar la planta física y mejorar las instalaciones existentes.

- Ampliar y fortalecer las relaciones del Centro con universidades nacionales e internacionales.

- Mantener la voz del Centro en eventos nacionales e internacionales sobre temáticas propias de la Educación, con propuestas innovadoras.

- Mejorar la capacitación y formación del personal académico y administrativo para garantizar calidad en el desarrollo de la academia.

- Mejorar el uso de la tecnología, tanto dentro de la academia como en el área administrativa, pues el Centro estaba muy retrasado en esa materia.

- Impactar, con propuestas concretas, el desarrollo de una educación de calidad en áreas de atención prioritaria, tanto en las zonas urbanas como rurales.

- $\quad$ Fortalecer el trabajo académico de las Divisiones para que lograran incorporar más proyectos de investigación y extensión.

- Fortalecer la docencia universitaria mediante acciones de investigación, capacitación, perfeccionamiento y producción académica.

- Mejorar las relaciones y la coordinación académica entre las escuelas que participaban en la formación de docentes y la División de Educología.

- $\quad$ Fortalecer acciones que garantizaran un mayor alcance de los programas y proyectos de vida estudiantil, de tal manera que los estudiantes tuvieran mayor acceso a un servicio de calidad y oportunidades de crecimiento personal y profesional.

\section{LOGROS}

- $\quad$ En este periodo, el CIDE alcanzó la máxima matrícula de su historia: más de 4500 estudiantes por semestre, especialmente en el momento en que se formaron docentes por medio del convenio con el MEP.

- Se abrió el primer posgrado: la Maestría en Educación con énfasis en aprendizaje del Inglés y, tiempo después, en la enseñanza del Español como segunda lengua.

- Se reestructuró el Centro, de acuerdo con los cambios estipulados en el nuevo Estatuto de ese entonces, y se hizo un intenso trabajo para que la comunidad educativa participara activamente en la definición de la misión, la visión y los objetivos del Centro, y se establecen las bases de un plan estratégico, acción que culminó el siguiente decano.

- Las economías generadas por la administración del Convenio con el MEP permitieron construir el nuevo edificio y remodelar el antiguo, infraestructura que fue inaugurada al inicio de la siguiente gestión.

- Se rediseñaron todos los planes de estudio para ofrecer planes trimestralizados, con sus respectivos planes de transición y tablas de equivalencia entre planes. 
- $\quad$ Se amplió y fortaleció, en el nivel de licenciatura, la formación de maestros de primaria, dado que se había estancado en Bachillerato.

- $\quad$ Se inició y se fortaleció la apertura de proyectos de investigación y extensión en las diferentes unidades académicas.

- Se fortaleció la cooperación internacional, gracias al aporte que dio la División de Educaron Rural, especialmente en la atención de escuelas unidocentes. Luego, se amplió la capacitación en el campo de la administración educativa, mediante acciones conjuntas de extensión entre la División de Educación Rural y la de Educación para el Trabajo.

- Las comunidades urbanas en riesgo social se vieron favorecidas con proyectos impulsados desde la División de Educación Básica, donde se trabajaron de manera integral la docencia, la extensión y la investigación.

- Se dieron a conocer las acciones de desarrollo del Centro en el campo de la formación docente en diferentes foros nacionales y en el extranjero: Panamá, Cuba, República Dominicana, Centroamérica y España.

- $\quad$ El Centro ofreció capacitación en docencia universitaria y formación docente a un numeroso grupo de académicos centroamericanos. Para realizar esa tarea, los docentes del CIDE visitaron diferentes universidades en el exterior, donde desarrollaron programas de capacitación o participaron en pasantías, congresos y talleres ofrecidos en Costa Rica.

- $\quad$ Se establecieron las normativas y los procedimientos necesarios para el desarrollo de planes conjuntos con las sedes regionales de la Universidad, lo que mejoró mucho la relaciones.

- Se crearon la normativa y los procedimientos requeridos, para facilitar el reconocimiento de estudios de las carreras de Educación Básica entre las universidades estatales formadoras de maestros.

- $\quad$ Se dejó negociado y listo el convenio con la Fundación Omar Dengo para la apertura del laboratorio de cómputo, acción que se concretó en la siguiente Decanatura.

- Se dio asesoría y se participó en la elaboración de la Maestría en Derechos Humanos y Educación para la Paz, que, originalmente, iba a ser desarrollada conjuntamente por el CIDE, la Facultad de Filosofía y Letras y la Universidad para la Paz; sin embargo, la administración siguiente no la consideró de interés, por lo que hoy tiene su sede en el IDELA .

- Se participó en el desarrollo del proyecto de Colegio Humanístico, aunque en la administración siguiente no se participó en el desarrollo de este proyecto.

- Se amplió la cobertura tecnológica para de las secretarias de las unidades académicas, con nuevo equipo de cómputo, que hiciera más eficiente su trabajo.

- Se logró que tanto el personal académico como administrativo participara, de manera comprometida, solidaria y leal, con las metas del Centro. Gracias a eso, se vivió un clima institucional que favorecía el trabajo en equipo y las buenas relaciones interpersonales. Quizá este fue el ingrediente que permitió hacer mucho, aunque no se contaba con el contenido presupuestario adecuado, pues su personal ofreció su trabajo desinteresadamente, incluso en momentos ajenos a su horario, y muchos funcionarios contribuyeron con recursos económicos propios al desarrollo de numerosas actividades.

- Se diversificó la oferta académico para los docentes de educación secundaria al abrir la carrera de Bachillerato y Licenciatura en Ciencias de la Educación con énfasis en Didáctica de... y el Bachillerato en Ciencias de la Educación con énfasis en Educación de Adultos, carreras que se ofrecieron en la sede central y fuera de esta.

- Se creó y fortaleció una oficina de atención a estudiantes que contribuyera a mejorar y ampliar proyectos que les brindaran servicios y mejoraran sus posibilidades de acceso 
a todas las oportunidades que ofrece la universidad en materia de becas, actividades deportivas y recreativas, servicios de salud y, sobre todo, información sobre trámites dentro de la universidad.

\section{DIFICULTADES}

La problemática presupuestaria que tenía la Universidad, le exigió al Centro tomar decisiones, escoger cuáles programas o proyectos priorizaba y tenían que esperar un mejor momento para ser ejecutados. Esto afectó, especialmente, a la Dirección de Desarrollo Profesional y Cooperación, encargada de investigar y capacitar a los docentes universitarios en Pedagogía Universitaria. Esta dirección operaba con personal prestado por diferentes unidades académicas, y la crisis de presupuesto las obligó a recuperar ese personal. Por esa razón, se dejó al descubierto dicha oferta; solo quedó una plaza para secretaria y otra para la Dirección, que fueron utilizadas para abrir el primer posgrado. En mi criterio, eso fue un desacierto, porque hubo que abandonar un área fundamental del quehacer del Centro, pues por medio del trabajo de esa Dirección se podía influir, en materia de educación, en otras facultades y centros.

Por otro lado, la coordinación entre la División de Educología y las otras unidades académicas, a pesar de los logros trascendentales obtenidos en el diseño de los cursos del componente pedagógico, no logró la unidad pretendida para desarrollar un currículo más integral, que garantizara una formación monolítica entre la escuela respectiva y la formación en Didáctica. En muchos casos, los problemas surgidos ante la necesidad de desarrollar los planes de estudio del Convenio, afectaron el desarrollo de acciones que contribuyeran a lograr esa integralidad; es decir, el problemas de los "feudos académicos" no logró resolverse realmente, pues la descalificación a la Educación prevalecía, a pesar de que la mayoría de los que la descalificaban vivían de ella, y eran empíricos en relación con los procesos de enseñanza.

Fue imposible editar la revista del CIDE, que necesitaba de presupuesto para su publicación y para contratar un director; la crisis presupuestaria imposibilitó el desarrollo de un proyecto importante y necesario, no solo para el Centro y la Universidad, sino para el país en general.

Se abortó el Instituto de la Juventud, organismo que concentraba la atención en la población adolescente y joven del país, porque algunos sectores de la comunidad académica consideraron que no era apto para el Centro, a pesar de que la juventud demandaba y sigue demandando una atención especial. Este proyecto fue adoptado posteriormente por la Facultad de Filosofía y Letras, donde se trabajó en pro del desarrollo de los jóvenes, especialmente de comunidades marginadas, que, en la actualidad, siguen siendo desatendidas y son la fuente de muchos de los problemas sociales en los inicios del siglo XXI.

Esta realidad muestra que la lucha tuvo como objetivo lograr que la Educación fuera un compromiso de todas y de todos. Se logró aportar, de manera importante y significativa, al desarrollo de la Educación, y madurar la misión de un Centro que siempre ha manifestado compromiso con el país, la universidad, los docentes y, sobre todo, con el mejoramiento de la calidad de educación que se les ofrece a las y los estudiantes, mediante procesos de enseñanza y aprendizaje de excelencia.

A pesar de las dificultades, y el poco interés de las autoridades universitarias por apoyar la educación como un compromiso de todos, la comunidad CIDE creció y dio sus aportes para contribuir a la excelencia en la formación de educadores. 


\section{REFERENCIAS}

Vargas, E. (1998). Informe de labores. Documento oficial no publicado. Heredia: Decanato-CIDE.

Universidad Nacional [UNA]. (1993). Estatuto orgánico de la Universidad Nacional. Heredia, Costa Rica. 